\title{
Article
}

\section{MRI Deep Learning-Based Solution for Alzheimer's Disease Prediction}

\author{
Cristina L. Saratxaga ${ }^{1, *}$, Iratxe Moya ${ }^{2}$, Artzai Picón ${ }^{1}{ }^{\infty}$, Marina Acosta ${ }^{2}$, Aitor Moreno-Fernandez-de-Leceta ${ }^{2}(\mathbb{D}$, \\ Estibaliz Garrote ${ }^{1,3}$ and Arantza Bereciartua-Perez ${ }^{1}$
}

1 TECNALIA, Basque Research and Technology Alliance (BRTA), Parque Tecnológico de Bizkaia, C/Geldo. Edificio 700, 48160 Derio, Spain; artzai.picon@tecnalia.com (A.P.); estibaliz.garrote@tecnalia.com (E.G.); aranzazu.bereciartua@tecnalia.com (A.B.-P.)

2 Instituto Ibermática de Innovación, Unidad de Inteligencia Artificial Avenida de los Huetos, Edificio Azucarera, 01010 Vitoria, Spain; i.moya@ibermatica.com (I.M.); m.acosta@ibermatica.com (M.A.); ai.moreno@ibermatica.com (A.M.-F.-d.-L.)

3 Department of Cell Biology and Histology, Faculty of Medicine and Dentistry, University of the Basque Country, 48940 Leioa, Spain

* Correspondence: cristina.lopez@tecnalia.com; Tel.: +34-946-430-850

Citation: Saratxaga, C.L.; Moya, I.; Picón, A.; Acosta, M.;

Moreno-Fernandez-de-Leceta, A.; Garrote, E.; Bereciartua-Perez, A. MRI Deep Learning-Based Solution for Alzheimer's Disease Prediction. J. Pers. Med. 2021, 11, 902. https:// doi.org/10.3390/jpm11090902

Academic Editor: Jorge Luis Espinoza

Received: 30 June 2021

Accepted: 2 September 2021

Published: 9 September 2021

Publisher's Note: MDPI stays neutral with regard to jurisdictional claims in published maps and institutional affiliations.

Copyright: (c) 2021 by the authors. Licensee MDPI, Basel, Switzerland. This article is an open access article distributed under the terms and conditions of the Creative Commons Attribution (CC BY) license (https:/ / creativecommons.org/licenses/by/ $4.0 /)$.

\begin{abstract}
Background: Alzheimer's is a degenerative dementing disorder that starts with a mild memory impairment and progresses to a total loss of mental and physical faculties. The sooner the diagnosis is made, the better for the patient, as preventive actions and treatment can be started. Although tests such as the Mini-Mental State Tests Examination are usually used for early identification, diagnosis relies on magnetic resonance imaging (MRI) brain analysis. Methods: Public initiatives such as the OASIS (Open Access Series of Imaging Studies) collection provide neuroimaging datasets openly available for research purposes. In this work, a new method based on deep learning and image processing techniques for MRI-based Alzheimer's diagnosis is proposed and compared with previous literature works. Results: Our method achieves a balance accuracy (BAC) up to 0.93 for image-based automated diagnosis of the disease, and a BAC of 0.88 for the establishment of the disease stage (healthy tissue, very mild and severe stage). Conclusions: Results obtained surpassed the state-of-the-art proposals using the OASIS collection. This demonstrates that deep learning-based strategies are an effective tool for building a robust solution for Alzheimer's-assisted diagnosis based on MRI data.
\end{abstract}

Keywords: deep learning; classification; Alzheimer's; MRI; OASIS

\section{Introduction}

Back in 1907, Alois Alzheimer described how a 51-year-old woman died with severe dementia after four years of rapid memory degeneration. Alzheimer's disease, named after him, is a dementia degenerative disease starting with mild memory impairment in the early stages and progressing to a complete loss of the mental and physical faculties [1]

The initial clinical manifestations of Alzheimer's disease (AD) are difficult to define as there is a large variation between cognitive abnormalities, but they can be correlated to degeneration in some specific regions of the brain. At first, AD typically presents memory loss and poor judgement. Then, the disease makes the patient more and more dependent, and in its later stages, he/she requires constant supervision. Although there is currently no cure for $\mathrm{AD}$, there is medication available that can temporarily reduce the symptoms, slowing down the progression of the disease and therefore, delaying the phase of complete dependence of the patient.

Over the years, different assessment tests have been proposed with the aim of diagnosing and monitoring the progress of AD.

The Mini-Mental State Examination (MMSE) exam [2], introduced in 1975, is the most extensively used questionnaire that examines six different modalities of patient cognitive 
ability. It is extensively used as it does not require special training or equipment and can be trustworthily used for the longitudinal monitoring of the AD progression. Unfortunately, its disadvantage is that it is affected by demographic, age and education factors and lacks sensitivity to measure progress in case of severe $\mathrm{AD}$. With a maximum of 30 points, results can be evaluated as normal cognition, mild, moderate and severe stages of the disease. Different score ranges for each category have been defined and discussed in the literature throughout the years, and it has been expanded accordingly in its current form [3].

The Clinical Dementia Rating (CDR) [4], introduced in 1982, is an alternative structured interview for measuring dementia based on the scoring obtained in six different cognitive and behavioral areas that are combined to obtain a value between 0 and 3 . The score values' meanings are as follows: 0 - cognitive normal, 0.5 — very mild or questionable dementia, 1-mild dementia, 2-moderate dementia and 3-severe dementia. An initial limitation was the difficulty to distinguish mild and very mild cases from normal cognition, so the test was updated later on to introduce new aspects that reliably distinguish those cases [5].

Other tests are the Clock Drawing test (CDT), which is the second most used test, although it lacks sensitivity for identifying early or moderate AD, the Alzheimer's Disease Assessment Scale (ADAS), the Global Dementia Scale (GDS) and the Neuropsychological Test Battery (NTB) [6].

However, definitive AD diagnosis relies on a magnetic resonance image (MRI) study [7]. MRI is a standard diagnosis technique free of radiation that is commonly used by healthcare providers for diagnosis, staging and follow-up of diseases. MRI scans are detailed three-dimensional anatomical images that can be used to examine almost any part of the body. In this case, the MRI technology makes it possible to recreate a 3D volume of the subject's head in detail [8]. The role of MRI in AD diagnosis is that it allows studying brain structures and how they change over the time. In this sense, changes in hippocampus, frontal and parietal regions are evidential markers in the progress of the disease to dementia [9]. Other imaging modalities such as PET (positron emission tomography) are also complementarily used to study changes in brain functioning and activity of key proteins. In recent years, the diagnosis process of the disease is evolving and initiatives based on various identified biomarkers have been proposed, with recommendations of the International Working Group on how they should be used in the clinical practice [10].

In this work, we propose a new approach for the automatic diagnosis of Alzheimer's disease by means of image processing and deep learning-based techniques using MRI data and CDR clinical annotation. Section 2 shows a review of available datasets and related work for comparison baseline definitions. Section 3 details the materials and methods used in this work and the proposed solution. Results are presented in Section 4 and discussion, conclusions and future research lines are presented in Section 5. Additionally, complementary information of materials and methods is included in Appendices A and B.

\section{Related Work}

\subsection{Data Collections}

ADNI (Alzheimer's Disease Neuroimaging Initiative) [11] is the most important and extensive collection of Alzheimer's-related information, containing different types of images, such as MRI, PET, DTI (diffusion tensor imaging), genetic information, demographic information, cognitive tests and cerebrospinal fluid (CSF) biomarkers from over 1800 subjects. The collection has been gathered based on different studies: ADNI1, ADNI-GO, ADNI2 and ADNI3, starting in 2010 and ongoing up to date. Imaging information has been acquired over different follow-ups with the aim of developing biomarkers to track the progression and underlying changes happening in the presence of AD. MRI images from different sequences (structural, diffusion-weighted, perfusion and resting state) and PET can be found in raw format or post-processed. Data science challenges, such as TADPOLE (The Alzheimer's Disease Prediction of Longitudinal Evolution) [12] in 2018, have made use of the ADNI collection, organizing the data in different standard sets containing longi- 
tudinal data (no longer available). Navigation in such a huge collection to obtain image data is a demanding task, and with the exception of the challenges, there are no standards sets that can be extensively used. For this reason, there are many research papers that use their own sets extracted from the original ADNI, making fair comparison of methodologies a very complex task, as reproductivity details to obtain a similar subset are not usually provided.

OASIS (Open Access Series of Imaging Studies) [13] is another well-known initiative that has provided neuroimaging datasets openly available for research purposes. Three noncomplementary, different datasets have been released over the years. These datasets are easily accessible, facilitating their usage and the validation of new proposed methodologies. OASIS-1 [14] (presented in 2007) is a cross-sectional collection of 416 subjects, where 100 are over 60 years of age and diagnosed with very mild or moderate Alzheimer's (using CDR). The dataset includes a T1-weighted MRI scan for each subject, with the exception of 20 subjects without dementia, scanned twice on a subsequent visit for baseline comparison, and a list of annotations. The MRI data is offered processed, with skull removal operation and segmentation masks separating grey-matter (GM), white-matter (WM) and CSF performed, and whole-brain volume and estimated total intracranial volume (eTIV) calculated. OASIS-2 [15] (presented in 2010) is a longitudinal collection of 150 subjects scanned on different visits, including a total of 373 imaging sessions. T1-weighted MRI scans and annotations are provided for each subject, plus calculations of the whole-brain volume presented, however, data processed with the skull removal and segmentation operation are not available. Interestingly, 14 of the subjects were initially identified as non-demented and converted to demented in a later visit (CDR reference). Additionally, OASIS-3 [16] (presented in 2019) is a longitudinal collection of 1090 subjects, 650 nondemented and 493 at various stages of AD. The dataset includes both MRI (structural and functional sequences) and PET (metabolic and amyloid) images, with over 2000 and 1500 sessions respectively, including some post-processing as segmentation and PET analyses, and annotations.

The ADCP (Alzheimer's Disease Connectome Project) [17] has gathered data from 300 subjects, cognitively healthy and with dementia, over a timespan of 4 years. Collected data includes standardized demographics, MRI (structural, function, diffusion-weighted) and PET (only for some participants) imaging, behavioral information and clinical information (blood tests including genetics and CSF proteins' analysis for 70\% of the participants). The data follow standards and protocols defined in the Connectome Project [18]. The collection has not been published yet, although it is planned to be released in two steps, first the baseline collection and later the longitudinal collection.

CADDementia (Computer-Aided Diagnosis of Dementia based on structural MRI data) [19] was an image processing challenge launched in 2014 containing 384 multi-center T1-weighted MRI images with AD, mild cognitive impairment (MCI) and healthy controls. A small training set (30 scans) with diagnosis labels was provided, although participants were encouraged to train on any suitable data (i.e., ADNI). The project provided a framework for the comparison of submitted computer-aided diagnosis methods.

The MIRIAD (Minimal Interval Resonance Imaging in Alzheimer's Disease) [20] dataset presented in 2013 included longitudinal volumetric T1 MRI scans of 46 subjects with mild-moderate AD and 23 controls, with a total of 798 scans acquired with the same equipment at established time intervals $(0,2,6,14,26,38$ and 52 weeks, and 18 and 24 months). Additional information is available, such as gender, age and MMSE scores.

The NACC (National Alzheimer's Coordinating Center) database [21] was released in 2007, presenting uniform data collected from 29 centers over 30 years. It contains more than 900 data elements grouped in different datasets and metadata with 68 data elements (such as race, education, gender, diagnosis, stroke, depression, availability of DNA, availability of tissue, availability of MRI, etc.). As suggested by the metadata, imaging information is not available for all users. Besides, access to the database is controlled via four users' types, from public to personnel restricted. 


\subsection{Automatic Analysis}

A systematic review of machine learning classification methods for assisted diagnosis is provided in [22], including literature works (conference and ArXiv are excluded) published during the years 2006-2016. Most works use traditional image processing methods for image pre-processing and feature extraction and traditional machine learning classification methods. Only 2 out of the 111 relevant studies considered were making use of a deep learning-based approach. Since 2016, as mentioned in [22], there has been a significant increase on the number of publications (with a big presence in conferences) using deep learning-based methods for the automatic classification of Alzheimer's disease on MRI images. In this sense, [23] provides the first review focused on deep learning (DL) methods, however, not only for AD classification but also for other brain-based disorders. The changes in the methodologies can also be clearly observed in the most recent review study [24], that includes works in the 2005-2019 period. The analysis is mainly organized in three categories, including support vector machines (SVM), artificial neural networks (ANN) and DL, although others are discussed. The problem of the clinical interpretability of DL models, usually seen as black boxes, in comparison with the other strategies, is addressed in the conclusions section.

Other works, such as [25], provide an alternative extensive review of methods and strategies up to 2016, where different image modalities are included, not only MRI. They include an interesting critical review of different aspects in the discussion section. First, they present aspects affecting works' comparison, such as the length of the follow-up period of patients in the datasets, characteristics of the population included, possible impairment in the data, evaluation metrics used and of other factors that can affect the performance of classification algorithms. Then, challenges still present on the AD classification works that are also common problems in the machine learning world, such as the ability to generalize, the size of datasets and samples, the reproductivity of the results and the heterogeneity of the AD disease, are presented.

In the current work, the authors have decided to use the OASIS collection datasets as baseline validation of the proposed methodology. The OASIS datasets' images and annotations are well-structured and organized, facilitating the reproductivity of the proposal and fair comparison of methods using exactly the same data. Other datasets provide more extensive data, but to the authors' knowledge, there are no standard subsets that can be used for fair comparison, hence works tent to create their own subset and details to facilitate reproductivity are usually missing. For this reason, from now on, analysis of the state-of-the-art has been focused only on those works making use of OASIS for comparison purposes, although there are additional methods developed over the ADNI or other databases with an extensive related literature. Besides, to be more precise and facilitate reading, only the most relevant and reproducible methods are mentioned or described in detail.

In general, there are two types of DL approaches: to combine Convolutional Neural Network $(\mathrm{CNN})$ with traditional image processing and machine learning methods (for feature selection and/or classification) or to develop full end-to-end CNN DL solutions. With respect to combined approaches, the most interesting approximations are found in [26-28]. References [26,27] report accuracies close to 99\%, whereas [28] reaches an $86.81 \%$ average accuracy for all classes. The reported accuracies with combined methods are impressive, however, in [26,27], few details are provided about the selection of cases in the dataset, meaning that the reported results can be biased and unrealistic. The proposal in [28] is interesting and reproducible, although no balanced accuracy (BAC) metric is provided, which is a more realistic metric to evaluate the accuracy of the approach in the presence of the high data imbalance of this dataset. Other metrics provided are accuracy, precision, recall and specificity, reporting $0 \%$ precision and recall for mild dementia and moderate $\mathrm{AD}$ classes due to the small number of cases in the dataset.

On the other side, with respect to full DL solutions, Reference [29], after testing different alternatives, proposed a 2D architecture which consists of an ensemble of three 
homogeneous and slightly different models containing convolutional, batch normalization, Rectified Linear Unit (ReLU) and pooling operations. For data augmentation, they proposed a cropping strategy, where three crops of size $112 \times 112$ pixels are extracted from each database sample, one from each image plane. They made use of the full OASIS-1 dataset, dedicating $70 \%$ for training, $10 \%$ for validation and $20 \%$ for testing. The model was trained independently and the 'softmax' classification layer with cross entropy was added to solve a four-class classification problem considering the CDR information on the dataset. A cost-sensitive training strategy is used for dealing with data imbalance, using a cost matrix to modify the output of the last layer of the networks for giving more importance to the underrepresented classes, assigning weights dependable to the number of samples of each class. Besides, models were optimized with the Stochastic Gradient Descent (SGD) [30] algorithm and early stopping regularization. Then, individual models' answers are ensembled using a majority voting strategy, where the class with the majority of the votes is assigned as the output answer. The mean accuracy of the proposed architecture is $93.18 \%$, with $94 \%$ precision ( $67 \%$ for mild dementia and $50 \%$ for moderate dementia) and $93 \%$ mean recall (33\% for very-mild dementia and $50 \%$ for moderate dementia).

More recently, Reference [31] provided a comprehensive review and comparison of methods proposed in the latest years. The authors mention the difficulties for fair comparison due to differences on participant selection, image pre-processing, model selection, validation procedure or the lack of implementation details (in more than half of the papers analyzed), which makes it difficult to reproduce the methods and could indicate biased performance of the reported metrics. They classified the identified approaches in four different groups considering the type of processing of the images: 2D slice-level, 3D patch-level, ROI-based and 3D subject level. Then, they have extended their open-source framework for the comparison of the selected CNN architectures, where they have detected that in general, neither of the proposals outperform an SVM with voxel-based features. As a result of their analysis, they provided different tables with the summaries of the studies analyzed and reported performances metrics (with data leakage identified) and the results of experiments implemented in their framework. In their experiments, they report the BAC metric and values obtained in five different executions (folds), comparing different classification architectures, training data configurations, image pre-processing strategies, intensity rescaling, training approaches, transfer learning approaches and classification tasks. For the OASIS collection (only patients over 62 years), they report average BAC within the range $[0.61,0.73]$ for the different experiments' configurations (details in Table 6 in [31]). The best performance reported in their experiments uses the ADNI dataset and reaches $0.89 \mathrm{BAC}$. This reference offers a valuable and fair framework for the comparison of the results of new proposals.

Considering the full DL solutions, possibly the most remarkable results are provided in [29], which makes use of 2D-based models ( 3 model resemble architecture), fair use of the complete OASIS-1 dataset, data augmentation and imbalance strategies and good learning practices, and they obtained 0.93 main accuracy in a four-class classification problem. However, the need of such architecture could be questionable as perhaps the results could be determined by the combination of ROIs from the 3 planes.

\section{Materials and Methods}

\subsection{Dataset}

This work makes use of the OASIS collection for the evaluation and comparison of the proposed methodology with previous works, and of the OASIS-1 and OASIS-2 datasets.

OASIS-1 [14] is a cross-section open dataset containing information from 416 subjects aged between 18 and 96 years, acquired with a $1.5 \mathrm{~T}$ scanner. Out of the 416 entries, 20 are from subjects without dementia, imaged in a subsequent additional visit later to their initial visit, as a control group for ensuring reliability of the data and analysis provided. All the subjects have right-hand dominance. 
The clinical status of the patient is established by the CDR scale, although the MMSE scale and other clinically relevant information along with demographic information is also provided for each entry. The annotation file contains the following information: ID, M/F, Hand, Age, Education (Educ), Socioeconomic status (SES), MMSE, CDR, estimated total intracranial volume (eTIV), normalized whole-brain volume (nWBV), atlas scaling factor (ASF) and Delay. 'Educ' indicates the years of education, SES uses the Hollinshead index of Social Position [32], MMSE score [2] and CDR scale [4] were established after medical examination, eTIV [33] and nWBV [34] were calculated as standard methods to analyze anatomical characteristics of the brain in the MRI images, ASF [33] was computed to transform the brain and skull from native space to the selected target atlas and 'Delay' for the 20 cases subgroup indicates the days after the first visit (mean: 20.55 days). The nWBV calculation requires a previous segmentation operation that separates WM, GM and CSF that has been performed based on estimations by the Markov random field model and further manual corrections.

Images of the OASIS-1 dataset are offered post-processed in 16-bit Analyze 7.5 format [35], with facial features out of the cranial values masked out, co-registered and converted to the Talairach and Tournoux standard atlas space [36] and inhomogeneity intensity due to the corrected magnetic field [37]. Additionally, different versions of the images are available: in RAW, processed without skull and segmented. In this work, we use processed images without skull, identified as "_t88_masked_gfc". This makes the OASIS-1 dataset able to be widely used. Nevertheless, out of these ideal dataset conditions, it is worth validating the methodology and performance of the models over other raw datasets.

OASIS-2 [15] is a longitudinal dataset of 150 subjects with ages between 60 and 96 years, scanned on various visits with a $1.5 \mathrm{~T}$ Vision scanner and at least one year difference, and a T1-weighted sequence, collecting a total of 373 imaging sessions. All the subjects have right-hand dominance. Interestingly, 14 of the subjects were characterized as non-demented on the initial visit and as demented afterwards, which can lead to studying the change on the whole-brain volume and structures to detect atrophy. Various subjects in the dataset were also part of the cross-sectional OASIS-1 (with different random identifiers), so the two datasets are not complementary.

The clinical status of the subjects is established by the CDR scale and contains the following annotations: Subject ID, MRI ID, Group, Visit, MR Delay, M/F, Hand, Age, EDUC, SES, MMSE, CDR, eTIV, nWBV and ASF. MRI ID identifies the number of the scan performed on the subject. 'Group' is a new annotation that classifies the subjects as 'Nondemented', 'Converted' or 'Demented', considering their status at the end of the study with respect to the beginning. Visit indicates the number of the visit of the entry and MR Delay indicates the number of days since the previous visit.

Images of the OASIS-2 dataset are offered converted to 16-bit NiFTI1 format, corrected for inter-scan head movement and converted to the Talairach and Tournoux standard atlas space [36] with a rigid transform. Images are co-registered with a 12-parameter affine transformation and intensity inhomogeneity, and variations among contiguous regions are corrected. Unfortunately, only the raw images are provided, and skull removal or segmentations are not available. This makes it difficult to directly use this dataset, requiring some pre-processing, which is explained in Appendix B.

The number of samples included in each dataset detailed by CDR diagnosis is summarized in Table 1. 
Table 1. Number of samples for the OASIS-1 and OASIS-2 datasets considering the CDR value as a reference.

\begin{tabular}{cccc}
\hline CDR & OASIS-1 & OASIS-2 & OASIS-2 (Our Subset) \\
\hline 0 (cognitive normal) & 336 & 206 & 177 \\
0.5 (very-mild dementia) & 70 & 123 & 98 \\
1 (mild dementia) & 28 & 41 & 27 \\
2 (moderate dementia) & 2 & 3 & 3 \\
\hline TOTAL & 436 & 373 & 305 \\
\hline
\end{tabular}

\subsection{Data and MRI Volume Processing: Model Input}

Images from both datasets have been labeled to face a classification problem. On the one hand, in two different classes, with subjects with $C D R=0$ being part of one class and subjects with $\mathrm{CDR}=0.5, \mathrm{CDR}=1$ or $\mathrm{CDR}=2$ part of the other class. The reason for this classification is that only 2 examples of $C D R=2$ and 28 of $C D R=1$ are part of the OASIS-1 dataset, being such an under-representation that it would lead to inaccurate multi-class classification results. On the other hand, they were grouped in a three-class problem (as explained above, there are not enough examples of CDR $=2$ for a four-class problem), where subjects with $C D R=0$ are part of one class, subjects with $C D R=0.5$ are part of another class and subjects with $\mathrm{CDR}=1$ and $\mathrm{CDR}=2$ are part of the remaining class.

All the available entries of the OASIS- 1 dataset are used in this work. From the OASIS2 dataset, some samples have been excluded due to skull removal problems, as explained in Appendix B and listed in Supplementary Table S1. No filtering per age (e.g., older than 60 years $[31,38]$ ) or other criteria has been performed in either of the datasets.

Training, validation and test sets are automatically randomly generated for the OASIS1 and OASIS-2 datasets, separately. Each set contains a balanced number of samples per sex $(\mathrm{M} / \mathrm{F})$ to eliminate possible bias. The reason is that previous studies state that incidence is different in women and men [39,40], and that differences in the brain volume-associated measures are observed for male subjects [41] with an impact on the prevalence of the disease. Additionally, for the OASIS-2 (longitudinal) dataset, the different entries (MRI scans in different visits) from the same subject are always included in the same set.

Only the horizontal (transverse) plane images have been used for training and testing the models. Some preliminary experiments were also performed using the frontal (coronal) plane images, but they were discarded as the results obtained were considered worse.

Datasets of MRI volumes have original dimensions of $176 \times 208 \times 176$, meaning that they contain 176 slices/images of $176 \times 208$ pixels size. Two different strategies have been considered in the experiments to include the MRI information. One strategy has been to use the whole 3D volume, which is especially interesting for models constructed with 3D convolutional layers. The other strategy has been to only use a limited number of slices of the volume, which has been demonstrated to be more interesting when experimenting with models built with 2D convolutional layers. Considering the center slice to be 88 (half of 176), an N number of slices from each side is extracted. Various experiments were executed with the same model with a different number of slices $(10,20,30$ and 50$)$ to conclude that the optimum value was 10 slices. Increasing the number of slices demonstrated that no improvements of the results were obtained, and even that using $\geq 30$ slices was unfavorable for the results. This suggests that the 10 center slices contain the most relevant information for diagnosis purposes. Then, original slices/images $(176 \times 208)$ have been resized to two different sizes depending on the model used, and implicit size restrictions if previous weights are used (e.g., ImageNet [42]). In this sense, two image sizes have been considered: $176 \times 176$ and $224 \times 224$ pixels.

Regardless of whether the model uses 2D or 3D convolutional layers, it can be trained with 3D data. In this sense, this work makes a comparison of 2D- and 3D-based architectures for the classification of Alzheimer's CDR and provides a comparison of results, including additional improvement strategies. Additionally, an approach that converts the 
3D data to 2D prior to training has also been implemented and included in the comparison. When data is converted to 2D, image slices are extracted from the 3D volume and treated independently during training, validation and testing. It is ensured that all image slices from the same sample are kept in the same data subset. Then, during the testing process, all images are evaluated independently, and a mean prediction is obtained (all images have the same weight), which determines the final prediction class.

Considering the data imbalance of the entries of the OASIS- 1 and OASIS-2 datasets (especially in the first case), the network generator is configured to always include the same number of samples of each class in the current batch, independently if targeting a two-class problem or a three-class problem. To increase variability and avoid overfitting, the data imbalance strategy is complemented with a data augmentation strategy, where images are randomly vertically or horizontally flipped or rotated. When the data are treated as 3D volume, the same operations are randomly applied to all the slices in the volume. On the contrary, when the data are treated as 2D, different operations can be applied in the data augmentation process to each of the individual images of the volume.

\subsubsection{Data Normalization}

Additionally, image values are normalized, and two strategies have been explored for image quality enhancement. In the basic form, 3D volume images are normalized to the [0-1] range. In an alternative configuration, and inspired by [43], a contrast stretching operation is performed. The $2 \mathrm{nd}$ and 98 th percentile values of the volume are calculated and assigned as min and max values, and then the whole volume is normalized to the [0-1] range.

\subsubsection{Metadata}

Following a similar approach to what is proposed in [28], it has been explored whether sex and age metadata can benefit model performance. As analyzed in Appendix A, from all the metadata available in the OASIS- 1 collection, sex and age information seem to have a relevant role in the diagnosis of Alzheimer's disease. Considering this, this information has been added to the MRI volume used as input (in the data generator) when training the models in some experiments for comparison purposes.

\subsection{Proposed Solution}

The aim of this work is to propose and develop a method to estimate the presence of Alzheimer's disease by means of the analysis of brain MRI sequence. As it was explained previously in the Introduction Section, the severity of Alzheimer's disease can be categorized through the CDR indicator used as a reference in the OASIS collection, whose value can be 0 (healthy), 0.5 (very mild), 1 (mild) or 2 (severe). Depending on the accuracy level, a two-, three- or four-class classification model can be developed. In this work, we have compared results of a two-class model and a three-class model, due to the CRD 1 and 2 categories containing fewer samples, so they have been combined.

The input of the model depends on the chosen architecture for the different tests. 2D (slice-level) and 3D (subject-level) approaches have been tackled. The 2D solution is usually used. The strongest point is that it allows to have a wider set of examples since there are 176 slices per MRI volume. This might allow a better training convergence and reduce overfitting. However, 2D approaches lack information in the third axis. This information when dealing with 3D objects, as it is the case with human tissues and organs, may provide details that will lead to a more precise solution [44].

For both 2D and 3D approaches, known architectures and custom architectures are considered. Among the known architectures, the ResNet [45] family has been evaluated, together with Inception [46], Xception [47], etc., with ResNet18 providing better results. Custom networks have been designed both in 2D convolution (Conv) and 3D convolution approaches. The implemented and compared network architectures are described next. 
BrainNet2D (2D Conv): A small custom network is proposed. The input data is considered as a 3D subject-level with single output for the whole volume. Input data are $\mathrm{M}$, $\mathrm{N}$ and $\mathrm{K}$, with $\mathrm{M}$ and $\mathrm{N}$ being the size of every slice, and $\mathrm{K}$ the number of slices. However, the information is treated slice-by-slice through the $2 \mathrm{D}$ convolutional layers and max pooling layers in order to reduce spatial resolution and extract a representative number of high-level descriptors. Four convolutional blocks are included, each of them containing the 2D convolutional layer (filter size is 3 ) and the 2D max pooling layer. No additional fully connected layers are added. The activation function is 'softmax' for exclusive classes. The loss function is initially 'categorical_crossentropy'. The architecture of BrainNet2D can be seen in Figure 1. Two variations of the same architecture are compared. First, the baseline BrainNet2D architecture (Figure 1A), and second, including Batch Normalization layers (Figure 1B) as an improvement technique, explained below.

A)

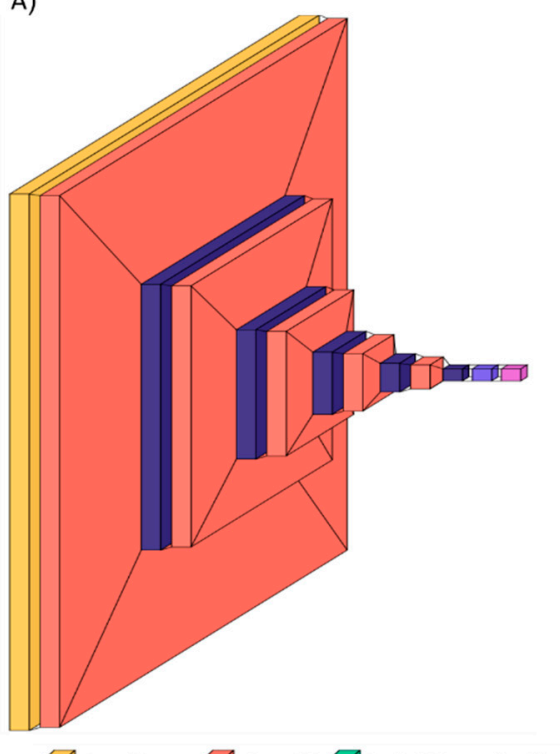

B)

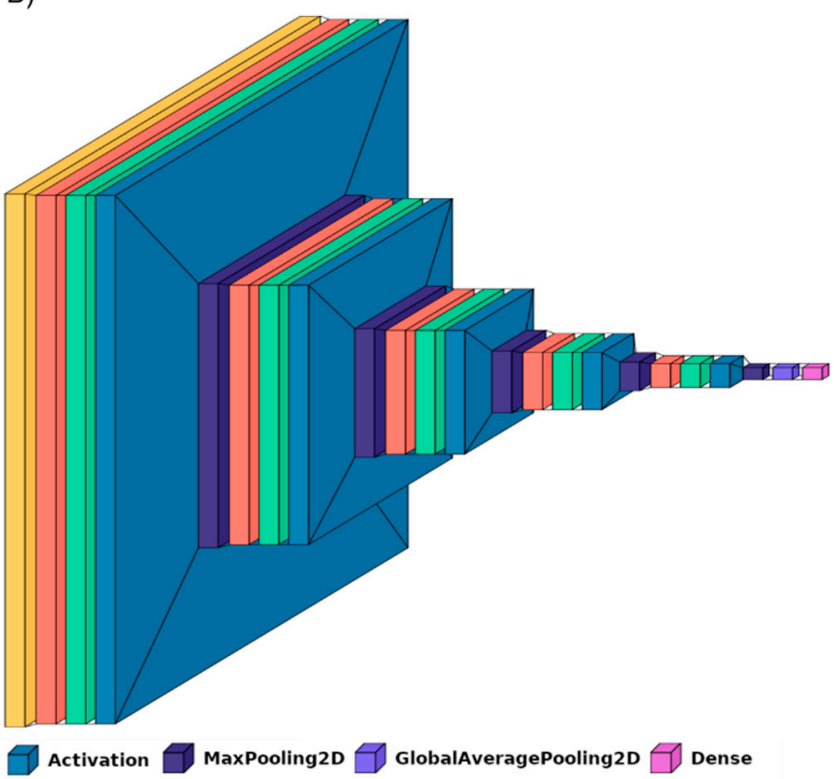

Figure 1. BrainNet2D network (A), and BrainNet2D network with Batch Normalization (B).

- $\quad$ BrainNet3D: A custom 3D network is proposed similar to the previous one but with a real 3D approach. Input data are $\mathrm{M}, \mathrm{N}, \mathrm{K}$ and 1, with $\mathrm{M}$ and $\mathrm{N}$ being the size of every slice, and $\mathrm{K}$ the number of slices. Five convolutional blocks are proposed, each of them containing the 3D convolutional layer and 3D max pooling layers. This approach fits better with biology and human understanding and might provide more useful patterns of structures in the brain. Filters for each convolutional layer represent the number of filters $*$ filter size, with the number of filters being 8, 16, 32, 64 and 128 in each of the five convolutional blocks, and the filter size is 3 in all of them. At the end of the embedding part, a Global Average Pooling 3D layer is added. No additional fully connected layers are added. The activation function is 'softmax' for exclusive classes. The loss function is initially 'categorical_crossentropy'. The architecture of BrainNet3D can be seen in Figure 1. Again, two variations of the same architecture are compared. First, the baseline BrainNet3D architecture (Figure 2A), and second, including the Batch Normalization layers as an improvement technique (Figure 2B). 
A)

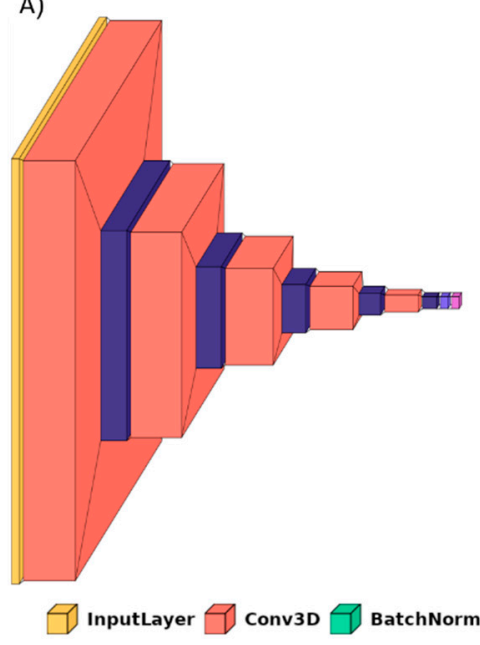

B)

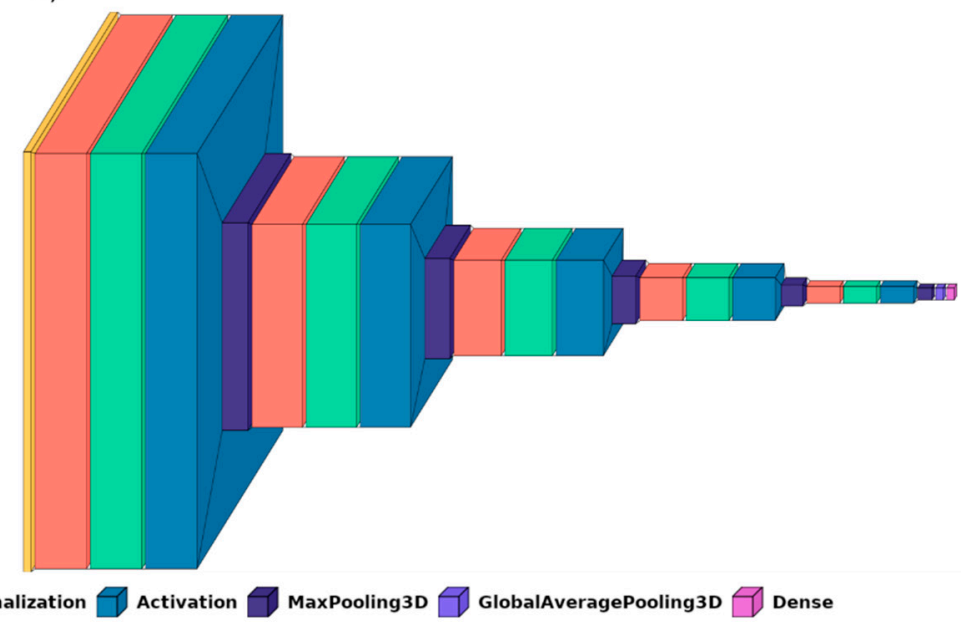

Figure 2. BrainNet3D network (A), and BrainNet3D network with Batch Normalization (B).

- 2D slice-level network: This network architecture performs at the 2D slice-level. This means that the network has as input a single slice of $\mathrm{M}, \mathrm{N}$ and 3 size, and every image has an associated classification output. The unique channel per slice has been replicated to provide the three-channel input expected by ResNet family networks and to make it possible to fine-tune operations with ImageNet weights. ResNet18, a small model from the ResNet family [45] pretrained on ImageNet, has been used as a reference model. After the embedding part, and as in the previous networks, the activation function is 'softmax' and the loss function is 'categorical_crossentropy'. In the testing phase, it must be pointed out that the final output for a complete study is provided in terms of 'majority vote' over all the slices of the sequence.

It is widely known by the people used to DL-based developments that there are some considerations in relation to the dataset and the specific details of the problem. If best practices are considered, the results may be improved [48]. The more remarkable strategies applied have been:

Cyclical Learning Rate: Cyclical Learning Rate (CLR) [49] is a strategy that allows oscillating between two learning rate values, iteratively. Previous traditional well-known learning strategies usually consider gradually decreasing the learning rate over the epoch using different functions (linear, polynomial, step, etc.). However, this strategy can lead the model to descend to areas of low loss values. With CLR, the optimal learning rate parameter can be easily found, and the model can consequently be better (and sooner) tuned. A minimum and a maximum learning rate value must be defined, and then the rate will cyclically oscillate between the two bounds. To do so, the different working policies can be defined: "triangular", which is a simple triangular cycle, "triangular2", also triangular but additionally cutting the maximum learning rate in half every cycle, and "exp_range", which is similar to the previous but with an exponential decay. Our experiments with CLR revealed that the triangular policy reported the best results with our datasets.

Batch Normalization: Batch Normalization [50] is a technique for training deep neural networks that standardizes the inputs to a layer for each mini-batch entering the network. This has the effect of stabilizing the learning process and often contributes to a better training process and thus better performance of the obtained model. Batch Normalization can also help to reduce overfitting, which is one of the main issues whenever a dataset is not large enough, as is the case with OASIS-1 and OASIS-2. This overfitting problem is mainly remarkable in the 3D-subject level approach. For the application of this Batch Normalization, the keras BatchNormalization() function, together with a 'relu' activation, is applied after the convolutional layer and before the max pooling layer. Figures 1 and 2 
illustrate how these layers have been integrated in the proposed BrainNet2D and BrainNet3D architectures, respectively.

Metadata: Sex and age have been revealed as relevant variables for the diagnosis, as concluded in Appendix A. Therefore, these two variables could facilitate the establishment of the classification output and have been incorporated in the network [48]. The inclusion in the network has been carried out after the embeddings and before the final activation layer. The metadata has been considered as follows: two classes for sex (male, female), and two classes for age $(<60$ and $>60)$.

ImageNet: Some experiments, particularly the ones adopting the ResNet18 architecture, have used pre-trained ImageNet weights. This practice often improves the results and accelerates the training process.

\section{Results}

The results obtained for the proposed methodology are shown in this section. First, results for classification models over the OASIS-1 dataset and the results obtained for the classification models over the OASIS-2 dataset are shown for the two-class problem (cognitive normal vs. AD). Then, results for the three-class problem (cognitive normal vs. very-mild dementia vs. mild and moderate dementia) using the OASIS-2 dataset are presented. Metrics used for the evaluation of classification models are accuracy (ACC) and balanced accuracy (BAC).

Different trainings were carried out over the OASIS- 1 dataset. Obtained results are shown in Table 2. All the experiments had common features that have not been included in the table. These common characteristics are the use of the horizontal planes of the 3D volume (coronal view), all the available MRI volumes have been used and the balanced inclusion of data according to sex has been also considered. These results correspond to the categorization of the input volume into healthy and with disease, i.e., a two-class classification problem. The table also shows the different parameters and operations that were used in the trainings, such as the normalization pre-processing action, experiment strategies (transfer learning, CLR triangular learning rate, Batch Normalization or the inclusion of metadata), the number of slices used in the process and the network image size.

Results obtained for the classification models over the OASIS-2 dataset (obtained as explained in Appendix B) for the two-class problem are shown in Table 3. The same common characteristics as for classification models in OASIS- 1 are applied here in all the trainings, however, experiments achieving worse results have been excluded from the comparison.

Table 2. Results for two-class classification, $C D R=0$ (healthy) and CDR $=0.5,1,2$ (disease), over the OASIS- 1 dataset.

\begin{tabular}{|c|c|c|c|c|c|c|c|}
\hline Network & Norm. & Strategies & Input Data & Slices Used & Image Size & Test ACC & Test BAC \\
\hline \multirow{4}{*}{ BrainNet2D } & {$[0,1]$} & CLR triangular & $3 \mathrm{D}$ & $\begin{array}{l}10 \text { (centered } \\
\text { in slice \#88) }\end{array}$ & 224 & $\begin{array}{l}\mathbf{0 . 8 1}[0.80 \\
0.80,0.80 \\
0.85,0.82]\end{array}$ & $\begin{array}{l}\mathbf{0 . 8 2}[0.81 \\
0.77,0.78 \\
0.86,0.86]\end{array}$ \\
\hline & $\begin{array}{l}\min -\max \\
\text { scaling }\end{array}$ & CLR triangular & $3 \mathrm{D}$ & $\begin{array}{l}10 \text { (centered } \\
\text { in slice \#88) }\end{array}$ & 224 & $\begin{array}{l}\mathbf{0 . 8 1}[0.83, \\
0.80,0.80 \\
0.82,0.80]\end{array}$ & $\begin{array}{l}\mathbf{0 . 8 1}[0.80 \\
0.77,0.77 \\
0.88,0.82]\end{array}$ \\
\hline & {$[0,1]$} & $\begin{array}{l}\text { CLR triangular } \\
\text { Batch Normalization }\end{array}$ & $3 \mathrm{D}$ & $\begin{array}{l}10 \text { (centered } \\
\text { in slice \#88) }\end{array}$ & 224 & $\begin{array}{l}\mathbf{0 . 7 9}[0.77, \\
0.78,0.86, \\
0.80,0.72]\end{array}$ & $\begin{array}{l}\mathbf{0 . 7 9}[0.77, \\
0.78,0.86, \\
0.80,0.72]\end{array}$ \\
\hline & {$[0,1]$} & $\begin{array}{l}\text { CLR triangular } \\
\text { Sex/Age metadata }\end{array}$ & $3 \mathrm{D}$ & $\begin{array}{l}10 \text { (centered } \\
\text { in slice \#88) }\end{array}$ & 224 & $\begin{array}{l}\mathbf{0 . 7 9}[0.80, \\
0.77,0.77 \\
0.88,0.82]\end{array}$ & $\begin{array}{l}\mathbf{0 . 8 4}[0.83 \\
0.85,0.85 \\
0.81,0.85]\end{array}$ \\
\hline
\end{tabular}


Table 2. Cont.

\begin{tabular}{|c|c|c|c|c|c|c|c|}
\hline Network & Norm. & Strategies & Input Data & Slices Used & Image Size & Test ACC & Test BAC \\
\hline \multirow[t]{4}{*}{ BrainNet3D } & $\begin{array}{l}\min -\max \\
\text { scaling }\end{array}$ & None & $3 \mathrm{D}$ & 176 (all) & 176 & $\begin{array}{l}\mathbf{0 . 7 8}[0.77, \\
0.78,0.80, \\
0.78,0.77]\end{array}$ & $\begin{array}{l}\mathbf{0 . 8 1}[0.83, \\
0.77,0.77, \\
0.82,0.85]\end{array}$ \\
\hline & $\begin{array}{l}\min -\max \\
\text { scaling }\end{array}$ & Batch Normalization & $3 \mathrm{D}$ & 176 (all) & 176 & $\begin{array}{l}\mathbf{0 . 7 9}[0.82, \\
0.80,0.78, \\
0.80,0.77]\end{array}$ & $\begin{array}{l}\mathbf{0 . 8 3}[0.85, \\
0.79,0.82, \\
0.86,0.83]\end{array}$ \\
\hline & $\begin{array}{l}\min -\max \\
\text { scaling }\end{array}$ & $\begin{array}{l}\text { Batch Normalization } \\
\text { Sex/Age metadata }\end{array}$ & $3 \mathrm{D}$ & 176 (all) & 176 & $\begin{array}{l}\mathbf{0 . 7 9}[0.78, \\
0.78,0.83, \\
0.80,0.78]\end{array}$ & $\begin{array}{l}\mathbf{0 . 8 2}[0.84, \\
0.77,0.82, \\
0.86,0.81]\end{array}$ \\
\hline & $\begin{array}{l}\min -\max \\
\text { scaling }\end{array}$ & $\begin{array}{l}\text { Batch Normalization } \\
\text { Sex/Age metadata } \\
\text { CLR triangular }\end{array}$ & $3 \mathrm{D}$ & 176 (all) & 176 & $\begin{array}{l}\mathbf{0 . 8 0}[0.80, \\
0.82,0.85, \\
0.82,0.74]\end{array}$ & $\begin{array}{l}\mathbf{0 . 8 4}[0.88, \\
0.80,0.88, \\
0.88,0.76]\end{array}$ \\
\hline \multirow{3}{*}{ ResNet18 } & {$[0,1]$} & ImageNet weights & $2 \mathrm{D}$ & $\begin{array}{l}10 \text { (centered } \\
\text { in slice \#88) }\end{array}$ & 224 & $\begin{array}{l}\mathbf{0 . 7 8}[0.80, \\
0.82,0.77, \\
0.75,0.75]\end{array}$ & $\begin{array}{l}\mathbf{0 . 7 9}[0.85, \\
0.79,0.67, \\
0.80,0.84]\end{array}$ \\
\hline & $\begin{array}{l}\min -\max \\
\text { scaling }\end{array}$ & ImageNet weights & $2 \mathrm{D}$ & $\begin{array}{l}10 \text { (centered } \\
\text { in slice \#88) }\end{array}$ & 224 & $\begin{array}{l}\mathbf{0 . 8 1}[0.80, \\
0.80,0.83 \\
0.80,0.82]\end{array}$ & $\begin{array}{l}\mathbf{0 . 8 2}[0.80, \\
0.80,0.83, \\
0.80,0.82]\end{array}$ \\
\hline & $\begin{array}{l}\min -\max \\
\text { scaling }\end{array}$ & $\begin{array}{l}\text { ImageNet weights } \\
\text { CLR triangular }\end{array}$ & $2 \mathrm{D}$ & $\begin{array}{l}10 \text { (centered } \\
\text { in slice \#88) }\end{array}$ & 224 & $\begin{array}{l}\mathbf{0 . 8 1}[0.82, \\
0.83,0.86, \\
0.75,0.77]\end{array}$ & $\begin{array}{l}\mathbf{0 . 8 3}[0.82, \\
0.81,0.86, \\
0.80,0.85]\end{array}$ \\
\hline
\end{tabular}

Table 3. Results for two classes classification, $\mathrm{CDR}=0$ (healthy), $\mathrm{CDR}=0.5,1,2$ (disease) over OASIS-2 dataset.

\begin{tabular}{|c|c|c|c|c|c|c|c|}
\hline Network & Norm. & Strategies & Input Data & Slices Used & Image Size & Test ACC & Test BAC \\
\hline \multirow[t]{2}{*}{ BrainNet2D } & {$[0,1]$} & CLR triangular & $3 \mathrm{D}$ & $\begin{array}{l}10 \text { (centered } \\
\text { in slice \#88) }\end{array}$ & 224 & $\begin{array}{l}\mathbf{0 . 9 2}[0.94 \\
1.00,0.92, \\
0.92,0.83]\end{array}$ & $\begin{array}{l}\mathbf{0 . 9 2}[0.93 \\
1.00,0.92 \\
0.92,0.92\end{array}$ \\
\hline & {$[0,1]$} & $\begin{array}{l}\text { CLR triangular } \\
\text { Sex/Age metadata }\end{array}$ & $3 \mathrm{D}$ & $\begin{array}{l}10 \text { (centered } \\
\text { in slice \#88) }\end{array}$ & 224 & $\begin{array}{l}\mathbf{0 . 8 2}[0.96 \\
0.93,0.92 \\
0.46,0.81]\end{array}$ & $\begin{array}{l}\mathbf{0 . 8 3}[0.96, \\
0.95,0.92, \\
0.50,0.80]\end{array}$ \\
\hline \multirow{4}{*}{ BrainNet3D } & $\begin{array}{l}\min -\max \\
\text { scaling }\end{array}$ & None & $3 \mathrm{D}$ & 176 (all) & 176 & $\begin{array}{l}\mathbf{0 . 6 7}[0.68 \\
0.80,0.73, \\
0.51,0.63]\end{array}$ & $\begin{array}{l}\mathbf{0 . 6 7}[0.68, \\
0.81,0.73, \\
0.52,0.64]\end{array}$ \\
\hline & $\begin{array}{l}\min -\max \\
\text { scaling }\end{array}$ & Batch Normalization & $3 \mathrm{D}$ & 176 (all) & 176 & $\begin{array}{l}\mathbf{0 . 8 4}[0.81, \\
1.00,0.78, \\
0.82,0.79]\end{array}$ & $\begin{array}{l}\mathbf{0 . 8 4}[0.81, \\
1.00,0.78, \\
0.83,0.77]\end{array}$ \\
\hline & $\begin{array}{l}\min -\max \\
\text { scaling }\end{array}$ & $\begin{array}{l}\text { Sex/Age metadata } \\
\text { Batch Normalization }\end{array}$ & $3 \mathrm{D}$ & 176 (all) & 176 & $\begin{array}{l}\mathbf{0 . 7 7}[0.70, \\
0.80,0.82, \\
0.77,0.77]\end{array}$ & $\begin{array}{l}\mathbf{0 . 7 8}[0.70 \\
0.81,0.82 \\
0.79,0.77]\end{array}$ \\
\hline & $\begin{array}{l}\min -\max \\
\text { scaling }\end{array}$ & $\begin{array}{l}\text { Batch Normalization } \\
\text { CLR triangular }\end{array}$ & $3 \mathrm{D}$ & 176 (all) & 176 & $\begin{array}{l}\mathbf{0 . 7 9}[0.70 \\
1.00,0.69 \\
0.87,0.69]\end{array}$ & $\begin{array}{l}\mathbf{0 . 7 9}[0.70, \\
1.00,0.68, \\
0.88,0.70]\end{array}$ \\
\hline \multirow[t]{3}{*}{ ResNet18 } & {$[0,1]$} & ImageNet weights & $2 \mathrm{D}$ & $\begin{array}{l}10 \text { (centered } \\
\text { in slice \#88) }\end{array}$ & 224 & $\begin{array}{l}\mathbf{0 . 9 2}[0.94, \\
1.00,0.94, \\
0.92,0.79]\end{array}$ & $\begin{array}{l}\mathbf{0 . 9 1}[0.94, \\
1.00,0.94, \\
0.92,0.77]\end{array}$ \\
\hline & {$[0,1]$} & $\begin{array}{l}\text { ImageNet weights } \\
\text { CLR triangular }\end{array}$ & $2 \mathrm{D}$ & $\begin{array}{l}10 \text { (centered } \\
\text { in slice \#88) }\end{array}$ & 224 & $\begin{array}{l}\mathbf{0 . 9 1}[0.94 \\
0.91,0.94 \\
0.95,0.83]\end{array}$ & $\begin{array}{l}\mathbf{0 . 9 2}[0.94, \\
0.94,0.94, \\
0.94,0.84]\end{array}$ \\
\hline & $\begin{array}{l}\min -\max \\
\text { scaling }\end{array}$ & $\begin{array}{l}\text { ImageNet weights } \\
\text { CLR triangular }\end{array}$ & $2 \mathrm{D}$ & $\begin{array}{l}10 \text { (centered } \\
\text { in slice \#88) }\end{array}$ & 224 & $\begin{array}{l}\mathbf{0 . 9 3}[0.94, \\
1.00,0.96, \\
0.92,0.83]\end{array}$ & $\begin{array}{l}\mathbf{0 . 9 3}[0.94, \\
1.00,0.96, \\
0.92,0.81]\end{array}$ \\
\hline
\end{tabular}

The BAC metric is the one that reflects the real accuracy weighted by class, so it is the metric value that, in the opinion of the authors, better reflects the performance of the 
model. BrainNet3D with min-max scaling normalization and Batch Normalization, or BrainNet2D with $[0,1]$ normalization, CLR triangular learning rate and the inclusion of the sex and age as metadata, seem to show slightly better results.

The experiments with the OASIS-2 dataset reveal improvements of the results for 2D network models. BrainNet2D- and ResNet18-based experiments provide BAC above 0.90 , which is clearly above the state-of-the-art for these datasets. The BrainNet2D approach) achieves a BAC up to 0.92 as the average BAC of a 5-fold experiment, and the RestNet18 approach () a BAC up to 0.93 .

BrainNet3D with Batch Normalization also increases its performance without the help of the metadata. However, the improvement rate is not as high as for $2 \mathrm{D}$ approaches. The possible reason for the poorer improvement may be that for the subject-level input approach, that is, the 3D approach, the good skull removal and pre-processing applied to all the slices in the sequences is required in the input. Minor movements in the registration and inaccurate pre-processing operation over the OASIS- 2 dataset performed by us, such as not perfect skull removal, may have led to incoherent and confusing input volumes for the 3D network. On the contrary, the 2D information of the slices is independent of the 3D volume-it does not matter whether there is good continuity on the z-axis. Every slice is dealt with separately, and the number of images is remarkably higher.

These additional experiments with the OASIS-2 dataset seem to reveal that the sex/age metadata are not a stable source of information to enrich the model during the classification task. The two experiments that included the metadata () achieved BAC values significantly inferior to the other experiments with the same model. This can be due to the fact that the metadata are highly biased by the characteristics of the population included in the datasets, but are not representative of the real clinical incidence of AD.

So far, a two-class problem has been addressed. Nevertheless, it is often useful to know the stage of the disease in case it is present. On that basis and relying on the bestperforming models obtained from the experiments over the OASIS-2 dataset, a three-class problem has been tested. The experiments have been launched only over the OASIS-2 dataset due to the under-represented mild and severe stages of the disease in the OASIS-1 dataset. The results obtained are gathered in Table 4.

Table 4. Results for three-class classification, $\mathrm{CDR}=0$ (healthy), $\mathrm{CDR}=0.5$ (very mild stage) and CDR $=1,2$ (severe stage), over the OASIS-2 dataset.

\begin{tabular}{|c|c|c|c|c|c|c|c|}
\hline Network & Norm. & Strategies & Input Data & Slices Used & Image Size & Test ACC & Test BAC \\
\hline BrainNet2D & {$[0,1]$} & CLR triangular & $3 \mathrm{D}$ & $\begin{array}{l}10 \text { (centered } \\
\text { in slice \#88) }\end{array}$ & 224 & $\begin{array}{l}\mathbf{0 . 8 8}[0.94 \\
1.00,0.78 \\
0.90,0.77]\end{array}$ & $\begin{array}{l}\mathbf{0 . 8 5}[0.94, \\
1.00,0.62, \\
0.92,0.76]\end{array}$ \\
\hline BrainNet3D & $\begin{array}{l}\min -\max \\
\text { scaling }\end{array}$ & Batch Normalization & $3 \mathrm{D}$ & 176 (all) & 176 & $\begin{array}{l}\mathbf{0 . 7 7}[0.87 \\
0.84,0.80 \\
0.74,0.58]\end{array}$ & $\begin{array}{l}\mathbf{0 . 7 6}[0.87 \\
0.85,0.63, \\
0.82,0.63]\end{array}$ \\
\hline ResNet18 & {$[0,1]$} & $\begin{array}{l}\text { ImageNet weights } \\
\text { CLR triangular }\end{array}$ & $2 \mathrm{D}$ & $\begin{array}{l}10 \text { (centered } \\
\text { in slice \#88) }\end{array}$ & 224 & $\begin{array}{l}\mathbf{0 . 8 9}[0.94 \\
0.98,0.84 \\
0.92,0.79]\end{array}$ & $\begin{array}{l}\mathbf{0 . 8 8}[0.94, \\
0.99,0.67 \\
0.94,0.85]\end{array}$ \\
\hline
\end{tabular}

These results again reveal that the $2 \mathrm{D}$ approach is more efficient than the $3 \mathrm{D}$ approach with subject-level input. BrainNet2D and ResNet18 solutions provide similar results, with the ones provided by ResNet18 being slightly better. These results point out that the methodology used is good to distinguish between mild Alzheimer's and severe stages of the disease. This is really valuable, since the sooner the disease is detected the better, in order to start more adequate treatment. 


\section{Discussion and Conclusions}

The present work provided main contributions as described below. First, we performed a systematic literature review on the topic, where limitations of the different works also using the OASIS collection were addressed. The main drawback is always data scarcity to facilitate comparisons. In general, DL-based techniques and Convolutional Neural Networks usually require a big dataset to be properly trained, and especially for the complex problems, as this is. OASIS-1 is a commonly used dataset in works dealing with Alzheimer's disease prediction. This dataset has strong points since it has well-registered and pre-processed sequences. Moreover, the skull is removed from the images and that ensures that the starting conditions for whatever study are the best. Masking for grey and white matters and cerebrospinal fluid is available, which makes it also useful to address segmentation problems. This is the reason why many works use this dataset in detriment of others that can provide a higher number of cases but that offer a poorly processed input, usually raw sequence data. Besides, the organization of the OASIS collection in different datasets, where images and annotations are easily accessible, facilitates the fair comparison of methods.

OASIS-2 has been used for validation purposes of the proposed approach in a wider set of studies than the perfectly registered and pre-processed OASIS-1. However, the sequences in this dataset are provided in raw format. This includes artefacts, noise and other elements in the head, such as the skull, that require processing before using. It is advisable to make the input data of OASIS-2 as similar as possible to the OASIS- 1 dataset. The main issue to be tackled is skull removal. In our work, a pre-processing pipeline has been proposed in Appendix B that aims at applying a similar pre-processing to the one semiautomatically performed in OASIS-1. In this way, additional OASIS-2 studies are available in the same conditions as OASIS-1 to be used in the same experiments. Unfortunately, both datasets could not be combined to improve model training since some of the subjects are repeated in both datasets and cannot be identified.

In this work, we proposed different approaches to tackle the problem: $2 \mathrm{D}$ and $3 \mathrm{D}$ networks in a slice-level approach or a subject-level approach. Custom networks (BrainNet2D, BrainNet3D) were proposed together with well-known architectures with transfer learning approaches such as fine-tuning with ImageNet weights. Modifications to the pre-processing have been applied to enhance the contrast of the grey-level structures in the brain. Different numbers of slices have been considered under the premise that it is not always the case that the higher the number of the images is, the better the results are. It might be true that it is worth providing a reduced number of valuable and meaningful slices instead of hundreds of slices that do not contain useful information.

Tables 2 and 3 showed results obtained for classification models over OASIS- 1 and OASIS-2 datasets for a two-class classification problem (cognitive normal or with Alzheimer's disease). Table 4 showed the results of a three-class (cognitive normal, very mild dementia, mild and moderate dementia) classification model that aims at obtaining higher precision in the detection of the stage of the disease. It can be shown that the presence of the disease can be established with a BAC of up to 0.93 with the OASIS-2 testing subset. In the three-class problem, additional stages in the disease can be predicted with a BAC of 0.88 .

A comparison of these results with state-of-the-art-methods considered reproducible and comparable is shown in Table 5. Reference [28] proposed a ResNet model-based approach for feature extraction, where age and sex metadata are added as additional features and a support vector machine classifier is used for a three-class classification, achieving a BAC of 0.86 . Reference [29] proposed a three-model ensemble using $112 \times 112$ crops input data from each anatomical plane and achieving an ACC of 0.93 for a three-class problem. Additionally, the authors of [31] performed a fair review of methods and strategies and their own implementation of them, reporting a BAC of 0.68 for a two-class problem using the OASIS dataset. 
Table 5. Comparison of the obtained results with the state-of-the-art methods that use the OASIS dataset for a twoclass classification problem: Cognitive normal and Alzheimer's disease. Comparison of results for a multiclass problem: Cognitive normal, Alzheimer's disease in very mild state and Alzheimer's disease in severe stage.

\begin{tabular}{|c|c|c|c|c|c|c|}
\hline \multirow[b]{2}{*}{ Method } & \multirow[b]{2}{*}{ Approach } & \multirow[b]{2}{*}{ Dataset } & \multicolumn{2}{|c|}{$\mathrm{CN}$ vs. $\mathrm{AD}$} & \multicolumn{2}{|c|}{$\begin{array}{c}\text { Multiclass: } \\
\text { CN vs. Mild vs. Severe }\end{array}$} \\
\hline & & & ACC & BAC & $\mathrm{ACC}$ & BAC \\
\hline $\begin{array}{l}\text { (PuenteCastro, } \\
\text { 2020) [28] }\end{array}$ & 2D slice level & OASIS-1 & - & - & - & 0.86 \\
\hline $\begin{array}{l}\text { (Islam and Zhang, } \\
\text { 2018) [29] }\end{array}$ & $\begin{array}{l}\text { 2D slice level } \\
(112 \times 112 \\
\text { crops })\end{array}$ & OASIS-1 & & - & 0.93 & - \\
\hline \multirow{2}{*}{ (Wen, 2020) [31] } & 2D slice level & $\begin{array}{l}\text { OASIS-1 (over } \\
62 \text { years) }\end{array}$ & - & $\begin{array}{l}0.68[0.68,0.67 \\
0.69,0.70,0.66]\end{array}$ & - & - \\
\hline & 3D subject level & & - & $\begin{array}{l}0.68[0.65,0.70 \\
0.70,0.71,0.65]\end{array}$ & - & - \\
\hline Our BrainNet2D & 2D slice level & OASIS-1 & $\begin{array}{l}\mathbf{0 . 7 9}[0.80,0.77 \\
0.77,0.88,0.82]\end{array}$ & $\begin{array}{l}\mathbf{0 . 8 4}[0.83,0.85 \\
0.85,0.81,0.85]\end{array}$ & & \\
\hline Out BrainNet3D & 3D subject level & OASIS-1 & $\begin{array}{l}\mathbf{0 . 8 0}[0.80,0.82 \\
0.85,0.82,0.74]\end{array}$ & $\begin{array}{l}\mathbf{0 . 8 4}[0.88,0.80 \\
0.88,0.88,0.76]\end{array}$ & & \\
\hline Our BrainNet2D & 2D slice level & OASIS-2 & $\begin{array}{l}\mathbf{0 . 8 2}[0.96,0.93 \\
0.92,0.46,0.81]\end{array}$ & $\begin{array}{l}\mathbf{0 . 8 3}[0.96,0.95 \\
0.92,0.50,0.80]\end{array}$ & $\begin{array}{l}\mathbf{0 . 8 8}[0.94,1.00 \\
0.78,0.90,0.77]\end{array}$ & $\begin{array}{l}\mathbf{0 . 8 5}[0.94,1.00, \\
0.62,0.92,0.76]\end{array}$ \\
\hline Out BrainNet3D & 3D subject level & OASIS-2 & $\begin{array}{l}\mathbf{0 . 8 4}[0.81,1.00 \\
0.78,0.82,0.79]\end{array}$ & $\begin{array}{l}\mathbf{0 . 8 4}[0.81,1.00 \\
0.78,0.83,0.77]\end{array}$ & $\begin{array}{l}\mathbf{0 . 7 7}[0.87,0.84 \\
0.80,0.74,0.58]\end{array}$ & $\begin{array}{l}\mathbf{0 . 7 6}[0.87,0.85, \\
0.63,0.82,0.63]\end{array}$ \\
\hline ResNet18 & 2D slice level & OASIS-2 & $\begin{array}{l}\mathbf{0 . 9 3}[0.94,1.00 \\
0.96,0.92,0.83]\end{array}$ & $\begin{array}{l}\mathbf{0 . 9 3}[0.94,1.00 \\
0.96,0.92,0.81]\end{array}$ & $\begin{array}{l}\mathbf{0 . 8 9}[0.94,0.98 \\
0.84,0.92,0.79]\end{array}$ & $\begin{array}{l}\mathbf{0 . 8 8}[0.94,0.99, \\
0.67,0.94,0.85]\end{array}$ \\
\hline
\end{tabular}

From the observation of the table, we can conclude that the results obtained with the proposed solution are equal to the state-of-the-art or beyond in the case of two-class classification. To the best of our knowledge, the good results not only derive from the network architectures, but they also depend on the adequate strategies that have been implemented. The inclusion of the OASIS-2 dataset for validation of the proposed methodology has forced us to work in the MRI raw volume processing. This processing methodology can be validated as good since it has managed to generate good enough MRI volumes to provide good performance of the models. There are few works addressing the multiclass problem, with $[28,29]$ being the most relevant ones. The results obtained by our method are very similar to the ones other authors have presented recently. We would like to point out that the metrics derived from our experiments are average metrics from 5 -fold experiments, and not all the works used these metrics, but they present the best results obtained here. There are some experiments in this 5-fold approach that outperformed the state-of-the-art results, but we prefer to show the results as they are with average metrics of different experiments with randomly chosen training, validation and testing sets. This provides an idea about the stability and repeatability of the proposed solution.

Future work clearly implies the use of additional datasets, such as ADNI, for comparative validation analysis and testing the generalization of the proposed approach. It is also desired to create a bigger combined dataset (from different sources) to increase the variability of the input samples of the different target classes, aiming to allow a better model generalization and reliable application to new unseen data. In this sense, a higher number of studies with high CDR will be useful, since they are currently under-represented. It would also be nice to dive deeper into three- and four-class problems to have the capability of predicting the different stages of the disease with high accuracy. This would lead to highlighting reliable diagnosis support tools that can aid in the definition of more accurate treatments and a hence impact the life expectancy of the patients. Other additional future research lines considered include studying the limits and weaknesses of the models in 
terms of accuracy and robustness [51], and adapting strategies such as multi-targeted backdoor [52], where models are led to misclassification using triggers on the data.

Supplementary Materials: The following are available online at https:/ / www.mdpi.com/article/10 .3390/jpm11090902/s1, Table S1: Summary of cases excluded from the OASIS-2 dataset.

Author Contributions: Conceptualization, C.L.S. and A.B.-P.; methodology, C.L.S. and A.B.-P.; software, C.L.S., A.B.-P. and I.M.; validation, A.P., A.M.-F.-d.-L. and E.G.; formal analysis, M.A., C.L.S. and I.M.; investigation, C.L.S., A.B.-P. and I.M.; resources, A.M.-F.-d.-L. and E.G.; data curation, C.L.S. and I.M.; writing—original draft preparation, C.L.S., A.B.-P. and I.M.; writing-review and editing, A.P., A.M.-F.-d.-L. and E.G.; visualization, C.L.S. and A.B.-P.; supervision, A.B.-P., A.P., A.M.-F.-d.-L. and E.G.; project administration, A.B.-P. and A.M.-F.-d.-L.; funding acquisition, A.M.-F.-d.-L. and E.G. All authors have read and agreed to the published version of the manuscript.

Funding: This work was partially supported by the SUPREME project. This project has received funding from the Basque Government's Industry Department HAZITEK program under agreement ZE-2019/00022. This research has also received funding from the Basque Government's Industry Department under the ELKARTEK program's project ONKOTOOLS under agreement KK-2020/00069.

Data Availability Statement: Data available in a publicly accessible repository that does not issue DOIs. OASIS (Open Access Series of Imaging Studies [13]).

Acknowledgments: OASIS: Cross-Sectional: Principal Investigators: D. Marcus, R, Buckner, J, Csernansky J. Morris; P50 AG05681, P01 AG03991, P01 AG026276, R01 AG021910, P20 MH071616, U24 RR021382. OASIS: Longitudinal: Principal Investigators: D. Marcus, R, Buckner, J. Csernansky, J. Morris; P50 AG05681, P01 AG03991, P01 AG026276, R01 AG021910, P20 MH071616, U24 RR021382.

Conflicts of Interest: The authors declare no conflict of interest.

\section{Appendix A. Statistical Analysis of Demographics and Clinical Information}

The OASIS dataset provides some additional information together with the MRI volumes. It is worth conducting statistical analysis of this data associated to each patient to find out whether there is some correlation among the variables. In case some conclusions are extracted from this analysis, the implied variables are to be considered in further experiments as metadata, that might contribute to a more accurate prediction of the model.

Statistical analysis is based on population segmentation. The objective is to subgroup the subjects considering the demographic and clinical information from OASIS- 1 , which has been already mentioned in Section 3.1.

Processing huge amounts of data to support decision processes is gaining increasing attention in corporate IT strategies [53]. Data science is the discipline used to gain valuable insights from data by mathematical and analytical models and applications. Data science projects can profit from project management and process methodologies that work as success factors [54]. However, strictly following a project methodology could be a challenge for data science teams. In this project, the CRISP-DM (CRoss Industry Standard Process for Data Mining) [55] methodology was used. In CRISP-DM, the data description forms the basis for the data quality verification. Then, a statistical analysis and exploration of the data is performed to gain insight about the underlying data generation process. The data should be described on a meta-level and by their statistical properties [56]. The requirements defined regarding the data quality are verifying the completeness (the expected feature values), outliers (the maximum number over "five sigma" gaussian distribution), extremes (the maximum number over "seven sigma" gaussian distribution) and consistency (the format of the data, calculated with the kurtosis and Skewness methods) of the data. Data that do not satisfy the expected conditions could be treated as anomalies and need to be evaluated manually or excluded automatically. To mitigate the risk of anchoring bias in the definition phase, discussing the requirements with a domain expert is advised [57]. After the data quality analysis in the CRISP-DM method, we must check the statistical power. The gathered data often includes, besides the predictive target, noise and unwanted features from other sources. To get a "gold standard" dataset, optimizing the statistical 
power and the predictive power, missing values, not consistent fields and special values could be imputed with a model readable value. Here, the wrong values are imputed by substituted model predictions [58].

The data-intensive approaches of machine learning and artificial intelligence bring together extensive datasets to feed into a utilitarian evaluation. The extensive data also shed light on the biases that exist in the current data and larger environment. In this work, the not supervised clustering method used in order to build a data hierarchy to generate a graph representation of the initial information solves the bias problem in the target field [59].

After performing the data quality and verifying phases, the completeness, outliers, extremes and consistency of the data, a data quality value of 0.89 has been obtained in the "gold standard dataset". Then, using the K-means algorithm with cosine distance, the population is segmented into the optimal number of clusters: five. Studying the distribution, it can be observed that the algorithm chooses gender $(\mathrm{M} / \mathrm{F})$, age, education (Educ), eTIV, SES and MMSE score as determining factors. As illustrated in Figure A1, this leaves a first cluster where subjects with a high SES and a low Educ predominate (cluster 0 ), a second where there are mostly women with low SES and high Educ (cluster 1) and a third (cluster 2) that is practically complementary to the second with women but with high SES and low Educ. In the fourth cluster (cluster 3), there are mostly male subjects with high eTIV, and the youngest female subjects are found in the last cluster (cluster 4).

Apparently, the most relevant information that can be inferred from this segmentation by joining it to the CDR of the individuals is the following: In cluster 4 , the mean value of CDR is 0 . In addition, in clusters 1 and 2, we found that for women, the mean value of CDR does not reach 0.5. This information seems to show that women are less likely to suffer from severe dementia, however previously documented clinical studies suggest that women are much more likely to suffer from AD than men [39,40].

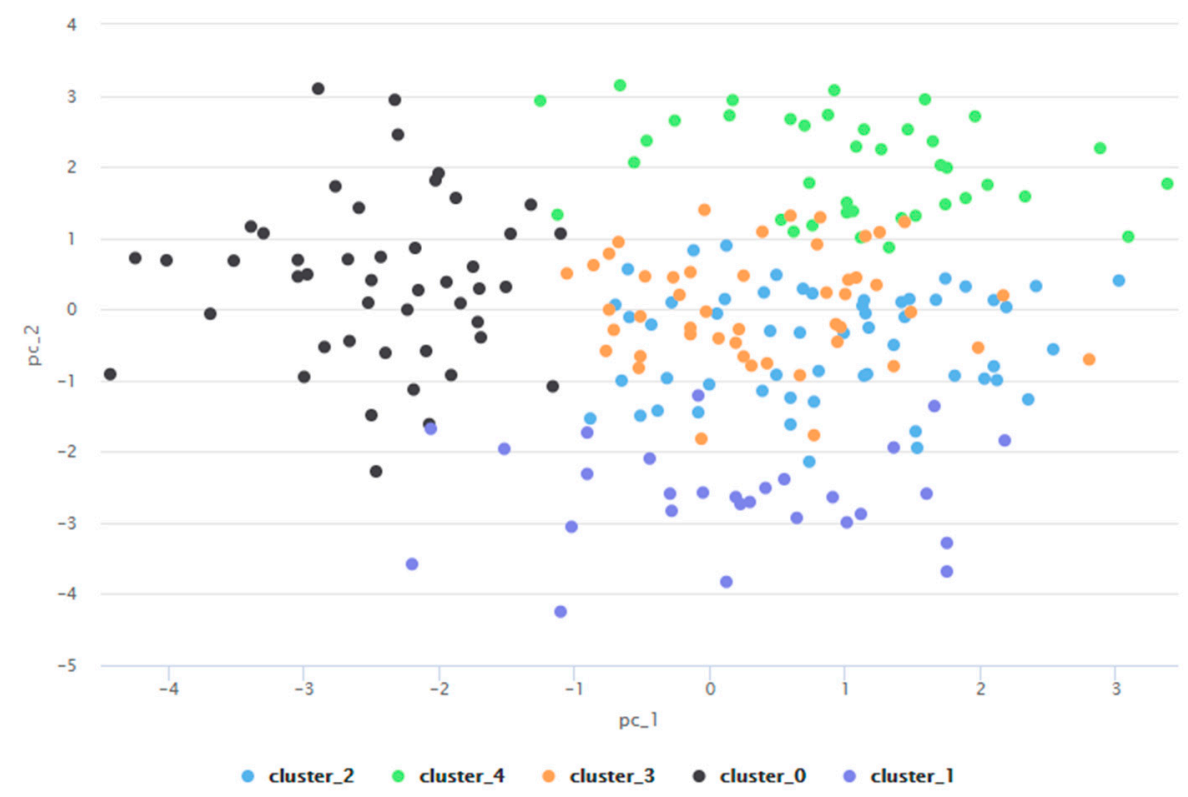

Figure A1. Distribution of the dataset into its two principal components separated in color by clusters.

\section{Appendix B. Pre-Processing of Raw MRI Volumes}

The pre-processing stage is a mandatory step in case the OASIS-2 dataset is aimed to be used to deal with the scarcity of data of OASIS- 1 and its class imbalance according to the CDR label. The aim is to replicate the pre-processing performed in OASIS- 1 with the OASIS-2 dataset, where only raw data is included. This pre-processing can be separated into two main steps: data preparation and skull removal. 
The aim of data preparation is to register and modify the display aspects of the volumes, such as voxel size or slice order. On the other hand, the skull removal process' purpose is to remove all those parts of the volume that are not strictly brain. The aim is to obtain the results illustrated in Figure A2, where the upper row shows the input raw data, and the bottom row shows the brain after the skull removal operation.

For data in the preparation step, a combination of AFNI (Analysis of Functional Neuro Images) [60] and FSL (the FMRIB Software Library) libraries is used [61].

AFNI is a leading software package for the analysis and visualization of multiple MRI modalities: anatomical data, functional MRI (fMRI) and diffusion-weighted data (DW). Although the software makes many functionalities available for the user, in the data preparation process, only 3 are used: flirt, to register the images, 3dRESAMPLE, to sample volumes with voxels of $1 \times 1 \times 1 \mathrm{~mm}$, and 3dAFNItoNIFTI, to convert the AFNI file type to ANALYZE (NIFTI).

FSL is a comprehensive library of structural and functional brain image analysis tools [61], that also includes innumerable functionalities, but only 3 are used in the process: fslmaths, which allows operations between images, such as media, etc. In the case of the pre-processing process, it is used to obtain the average of all the registered images. Fslswapdim, which is used to change the order of the displayed dimensions of the volumes, and fslchfiletype, to change the file type, so that we can obtain ANALYZE images from NIFTI images.

The overall pre-processing process implemented can be described by the following steps:

1. Image registration, using the Flirt tool from the AFNI library: all images included in the RAW folder of the OASIS-2 database are registered in pairs.

2. Averaging registered images, using the fslmaths tool from the FSL library: all resultant images from the registration process are averaged to obtain one unique image.

3. Image resampling, using the 3dRESAMPLE tool from the AFNI library: the resultant averaged image is resampled to obtain $1 \times 1 \times 1 \mathrm{~mm}$ voxels.

4. Change volume slice order, using the fslswapdim tool from the FSL library: As original images do not have the same slice anatomical plane view as the pre-processed volumes in OASIS- 1 and the aim of the pre-processing part is to replicate as well as possible the pre-processing performed in OASIS-1, the dimension slices of the resampled image are swapped.

Next, the skull removal process is carried out using the BET2 (Brain Extraction Tool v2.0) [62] tool within the FLS library. BET2 is a fast and fully automated tool for extracting brain, inner and outer skull and scalp surfaces from MRI. It uses high-resolution T1 and $\mathrm{T} 2$ images, but it can be run only with $\mathrm{T} 1$ images, obtaining less accurate results. BET2 is based on the assumption that, generally, in MRI images, the skull appears darker than the rest of the tissues. In T1 images, CSF tissue is also dark, so it can be confused with the skull. In T2 images, on the other hand, CSF is bright, but muscles are often dark. These intensities can vary between different scans, but in T1 images, CSF will always be darker than the rest of the tissues, and in T2 images, muscles will always be darker, so by combining both images, brain tissue can be distinguished from the skull and the void (or air) more easily.

The process to achieve this is as follows. First, the outer scalp point is found, by combining the outermost non-dark T1 point and the outermost non-dark T2 point (thresholds are determined from robust histograms). Next, the inner skull point is found as a combination of searches (outwards from the brain) for the "first dark" T1 and T2 points. As due to topological complications in certain regions the outer skull point is harder to find robustly, a more complex combination of the T2 and T1 profiles is used to identify the most likely point. This approach correctly detects the outer skull points in much of the head, but fails in the lower areas, where tissues and skull topology are too complex, so an alternative algorithm that takes into account some prior spatial knowledge is used in this part. The determination of the area where the alternative algorithm is needed is performed manually. Then, Flirt is used so that a standard space transform matrix is obtained, and this mask 
is transformed into the space of the data. Then, the outer skin and inner skull points are obtained. If the outer skull point is too far from the inner skull point (about $7 \mathrm{~mm}$ ), the assumption is simplified to the fact that the outer skull point is $0.5 \mathrm{~mm}$ outside the inner skull point. Then, an image is created using the points obtained.

As said before, although using only T1 images is possible in BET2, the accuracy of the results is lower than using both T1 and T2 images. Nevertheless, the OASIS database only includes T1 images, so a various step process is needed to obtain good results in skull stripping. For the OASIS-2 non-processes dataset, first, BET2 is executed with a low fractional intensity threshold and eye and optic nerve clean-up option. Then, the results are visualized (using the fsleyes tool from FSL) and another BET2 execution is performed. This second time, the fractional intensity threshold varies in each volume (depending on the previous results) and, in some volumes, the bias field and neck clean-up option is activated in order to obtain better results. This visualization part is necessary as the second execution is a manual process. As the results of the output of the first execution depend on each volume, it is not possible to generalize the second execution for all the cases, and a manual evaluation of required parameters in the second execution is necessary. With this operation combination, it is possible to extract not only the skull but also all those tissues that are not brain, such as the optic nerve or the neck. However, in some volumes included in the OASIS-2 original database, the skull stripping process was not successful because of the quality of the T1 image or the low-intensity difference between CSF and the skull. For this reason, these volumes were excluded from OASIS-2 datasets for the experiments. The identifiers of the volumes excluded are described in Supplementary Table S1 to facilitate the reproductivity of the results presented in this work.

Following the previously described process, the pre-processing performed in OASIS-1 has been replicated, and pre-processed volumes for OASIS-2 have been created in order to augment the amount of data for training and testing the proposed approach. The results of this process are illustrated in the sample included in Figure A2.

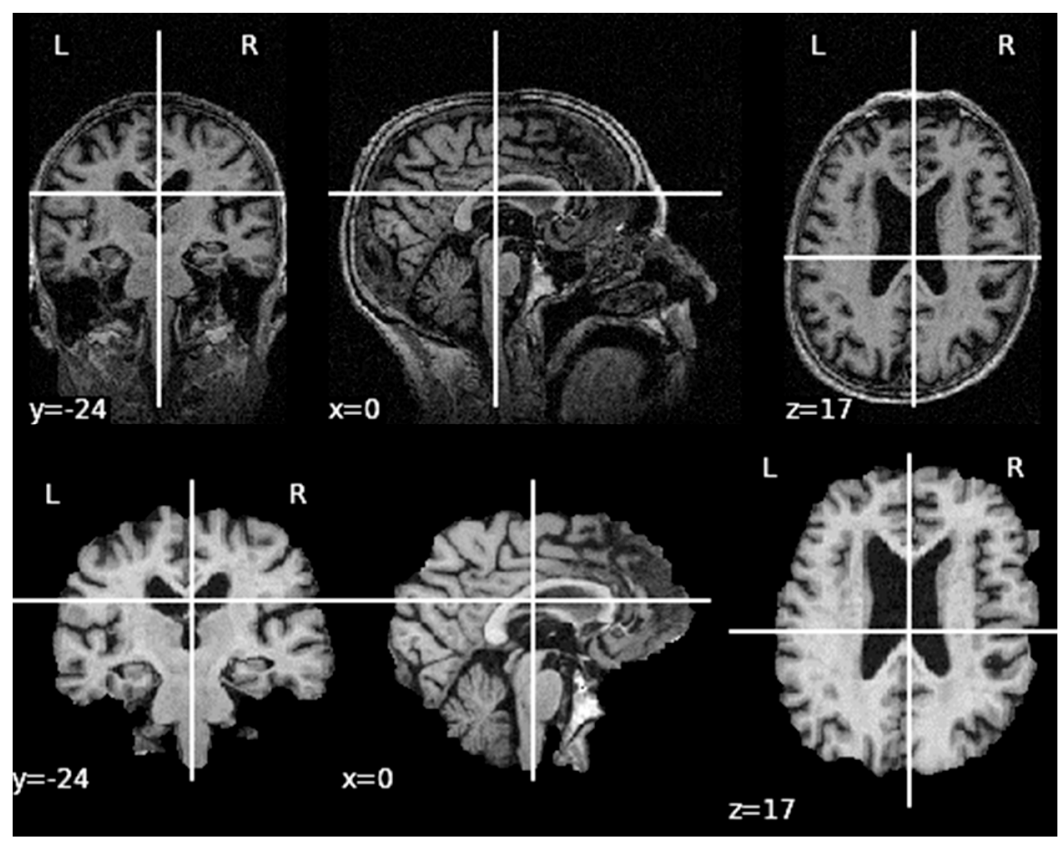

Figure A2. MRI volume prior to processing (upper row) and after skull removal processing (bottom row). Column A: coronal plane, column B: sagittal view and column C: axial plane. 


\section{References}

1. Smith, M.A. Alzheimer disease. Int. Rev. Neurobiol. 1998, 42, 1-54. [CrossRef]

2. Folstein, M.F.; Folstein, S.E.; McHugh, P.R. 'Mini-mental state'. A practical method for grading the cognitive state of patients for the clinician. J. Psychiatr. Res. 1975, 12, 189-198. [CrossRef]

3. Mini-Mental State Examination Second Edition I MMSE-2. Available online: https://www.parinc.com/Products/Pkey/238 (accessed on 24 March 2021).

4. Hughes, C.P.; Berg, L.; Danziger, W.L.; Coben, L.A.; Martin, R.L. A new clinical scale for the staging of dementia. Br. J. Psychiatry 1982, 140, 566-572. [CrossRef] [PubMed]

5. Duara, R.; Loewenstein, D.A.; Greig-Custo, M.T.; Raj, A.; Barker, W.; Potter, E.; Schofield, E.; Small, B.; Schinka, J.; Wu, Y.; et al. Diagnosis and staging of mild cognitive impairment, using a modification of the clinical dementia rating scale: The mCDR. Int. J. Geriatr. Psychiatry 2010, 25, 282-289. [CrossRef] [PubMed]

6. Khan, T.K. Clinical Diagnosis of Alzheimer's Disease. In Biomarkers in Alzheimer's Disease; Elsevier: Amsterdam, The Netherlands, 2016; pp. 27-48.

7. Frisoni, G.B.; Fox, N.C.; Jack, C.R.; Scheltens, P.; Thompson, P.M. The clinical use of structural MRI in Alzheimer disease. Nat. Rev. Neurol. 2010, 6, 67-77. [CrossRef] [PubMed]

8. McRobbie, D.W.; Moore, E.A.; Graves, M.J.; Prince, M.R. MRI from Picture to Proton, 3rd ed.; Cambridge University Press: Cambridge, UK, 2017; ISBN 9781107643239.

9. Corriveau-Lecavalier, N.; Mellah, S.; Clément, F.; Belleville, S. Evidence of parietal hyperactivation in individuals with mild cognitive impairment who progressed to dementia: A longitudinal fMRI study. NeuroImage Clin. 2019, 24, 101958. [CrossRef]

10. Dubois, B.; Villain, N.; Frisoni, G.B.; Rabinovici, G.D.; Sabbagh, M.; Cappa, S.; Bejanin, A.; Bombois, S.; Epelbaum, S.; Teichmann, M.; et al. Clinical diagnosis of Alzheimer's disease: Recommendations of the International Working Group. Lancet Neurol. 2021, 20, 484-496. [CrossRef]

11. ADNI | Alzheimer's Disease Neuroimaging Initiative. Available online: http://adni.loni.usc.edu/ (accessed on 23 March 2021).

12. Marinescu, R.V.; Oxtoby, N.P.; Young, A.L.; Bron, E.E.; Toga, A.W.; Weiner, M.W.; Barkhof, F.; Fox, N.C.; Klein, S.; Alexander, D.C. TADPOLE challenge: Prediction of longitudinal evolution in Alzheimer's disease. arXiv 2018, arXiv:1805.03909.

13. OASIS Brains-Open Access Series of Imaging Studies. Available online: https://www.oasis-brains.org/ (accessed on 23 March 2021).

14. Marcus, D.S.; Wang, T.H.; Parker, J.; Csernansky, J.G.; Morris, J.C.; Buckner, R.L. Open Access Series of Imaging Studies (OASIS): Cross-sectional MRI data in young, middle aged, nondemented, and demented older adults. J. Cogn. Neurosci. 2007, 19, $1498-1507$. [CrossRef]

15. Marcus, D.S.; Fotenos, A.F.; Csernansky, J.G.; Morris, J.C.; Buckner, R.L. Open access series of imaging studies: Longitudinal MRI data in nondemented and demented older adults. J. Cogn. Neurosci. 2010, 22, 2677-2684. [CrossRef]

16. LaMontagne, P.J.; Benzinger, T.L.S.; Morris, J.C.; Keefe, S.; Hornbeck, R.; Xiong, C.; Grant, E.; Hassenstab, J.; Moulder, K.; Vlassenko, A.G.; et al. OASIS-3: Longitudinal neuroimaging, clinical, and cognitive dataset for normal aging and Alzheimer disease. medRxiv 2019, 12, 13. [CrossRef]

17. Alzheimer's Disease Connectome Project. Available online: https://www.humanconnectome.org/study/alzheimers-diseaseconnectome-project (accessed on 24 March 2021).

18. Connectome-Homepage. Available online: https://www.humanconnectome.org/ (accessed on 24 March 2021).

19. Bron, E.E.; Smits, M.; van der Flier, W.M.; Vrenken, H.; Barkhof, F.; Scheltens, P.; Papma, J.M.; Steketee, R.M.E.; Méndez Orellana, C.; Meijboom, R.; et al. Standardized evaluation of algorithms for computer-aided diagnosis of dementia based on structural MRI: The CADDementia challenge. Neuroimage 2015, 111, 562-579. [CrossRef]

20. Malone, I.B.; Cash, D.; Ridgway, G.R.; MacManus, D.G.; Ourselin, S.; Fox, N.C.; Schott, J.M. MIRIAD-Public release of a multiple time point Alzheimer's MR imaging dataset. Neuroimage 2013, 70, 33-36. [CrossRef] [PubMed]

21. Beekly, D.L.; Ramos, E.M.; Lee, W.W.; Deitrich, W.D.; Jacka, M.E.; Wu, J.; Hubbard, J.L.; Koepsell, T.D.; Morris, J.C.; Kukull, W.A.; et al. The National Alzheimer's Coordinating Center (NACC) database: The uniform data set. Alzheimer Dis. Assoc. Disord. 2007, 21, 249-258. [CrossRef] [PubMed]

22. Pellegrini, E.; Ballerini, L.; Hernandez, M.D.C.V.; Chappell, F.M.; González-Castro, V.; Anblagan, D.; Danso, S.; Muñoz-Maniega, S.; Job, D.; Pernet, C.; et al. Machine learning of neuroimaging for assisted diagnosis of cognitive impairment and dementia: A systematic review. Alzheimer's Dement. Diagnosis, Assess. Dis. Monit. 2018, 10, 519-535. [CrossRef]

23. Vieira, S.; Pinaya, W.H.L.; Mechelli, A. Using deep learning to investigate the neuroimaging correlates of psychiatric and neurological disorders: Methods and applications. Neurosci. Biobehav. Rev. 2017, 74, 58-75. [CrossRef]

24. Tanveer, M.; Richhariya, B.; Khan, R.U.; Rashid, A.H.; Khanna, P.; Prasad, M.; Lin, C.T. Machine Learning Techniques for the Diagnosis of Alzheimer's Disease. ACM Trans. Multimed. Comput. Commun. Appl. 2020, 16, 1-35. [CrossRef]

25. Rathore, S.; Habes, M.; Iftikhar, M.A.; Shacklett, A.; Davatzikos, C. A review on neuroimaging-based classification studies and associated feature extraction methods for Alzheimer's disease and its prodromal stages. Neuroimage 2017, 155, 530-548. [CrossRef]

26. Khagi, B.; Kwon, G.; Lama, R. Comparative analysis of Alzheimer's disease classification by CDR level using CNN, feature selection, and machine-learning techniques. Int. J. Imaging Syst. Technol. 2019, 29, 297-310. [CrossRef] 
27. Nawaz, H.; Maqsood, M.; Afzal, S.; Aadil, F.; Mehmood, I.; Rho, S. A deep feature-based real-time system for Alzheimer disease stage detection. Multimed. Tools Appl. 2020,1-19. [CrossRef]

28. Puente-Castro, A.; Fernandez-Blanco, E.; Pazos, A.; Munteanu, C.R. Automatic assessment of Alzheimer's disease diagnosis based on deep learning techniques. Comput. Biol. Med. 2020, 120, 103764. [CrossRef]

29. Islam, J.; Zhang, Y. Brain MRI analysis for Alzheimer's disease diagnosis using an ensemble system of deep convolutional neural networks. Brain Inform. 2018, 5, 1-14. [CrossRef] [PubMed]

30. Bottou, L. On-line Learning and Stochastic Approximations. In On-Line Learning in Neural Networks; Cambridge University Press: Cambridge, UK, 2010; pp. 9-42.

31. Wen, J.; Thibeau-Sutre, E.; Diaz-Melo, M.; Samper-González, J.; Routier, A.; Bottani, S.; Dormont, D.; Durrleman, S.; Burgos, N.; Colliot, O. Convolutional neural networks for classification of Alzheimer's disease: Overview and reproducible evaluation. Med. Image Anal. 2020, 63, 101694. [CrossRef] [PubMed]

32. Hollingshead, A.B. Two Factor Index of Social Position; Yale University Press: New Haven, CT, USA, 1957.

33. Buckner, R.L.; Head, D.; Parker, J.; Fotenos, A.F.; Marcus, D.; Morris, J.C.; Snyder, A.Z. A unified approach for morphometric and functional data analysis in young, old, and demented adults using automated atlas-based head size normalization: Reliability and validation against manual measurement of total intracranial volume. Neuroimage 2004, 23, 724-738. [CrossRef]

34. Fotenos, A.F.; Snyder, A.Z.; Girton, L.E.; Morris, J.C.; Buckner, R.L. Normative estimates of cross-sectional and longitudinal brain volume decline in aging and AD. Neurology 2005, 64, 1032-1039. [CrossRef] [PubMed]

35. Larobina, M.; Murino, L. Medical Image File Formats. J. Digit. Imaging 2014, 27, 200-206. [CrossRef]

36. Talairach, J.; Tournoux, P. Co-Planar Stereotaxic Atlas of the Human Brain; Thieme: Stuttgart, Germany, 1988.

37. Styner, M. Parametric estimate of intensity inhomogeneities applied to MRI. IEEE Trans. Med. Imaging 2000, 19, 153-165. [CrossRef] [PubMed]

38. Suh, C.H.; Shim, W.H.; Kim, S.J.; Roh, J.H.; Lee, J.H.; Kim, M.J.; Park, S.; Jung, W.; Sung, J.; Jahng, G.H. Development and validation of a deep learning-based automatic brain segmentation and classification algorithm for Alzheimer disease using $3 \mathrm{D}$ T1-weighted volumetric images. Am. J. Neuroradiol. 2020, 41, 2227-2234. [CrossRef]

39. Filon, J.R.; Intorcia, A.J.; Sue, L.I.; Vazquez Arreola, E.; Wilson, J.; Davis, K.J.; Sabbagh, M.N.; Belden, C.M.; Caselli, R.J.; Adler, C.H.; et al. Gender differences in Alzheimer disease: Brain atrophy, histopathology burden, and cognition. J. Neuropathol. Exp. Neurol. 2016, 75, 748-754. [CrossRef] [PubMed]

40. Niu, H.; Álvarez-Álvarez, I.; Guillén-Grima, F.; Aguinaga-Ontoso, I. Prevalence and incidence of Alzheimer's disease in Europe: A meta-analysis. Neurology (Engl. Ed.) 2017, 32, 523-532. [CrossRef]

41. Whitwell, J.L. The protective role of brain size in Alzheimer's disease. Expert Rev. Neurother. 2010, 10, 1799-1801. [CrossRef]

42. Russakovsky, O.; Deng, J.; Su, H.; Krause, J.; Satheesh, S.; Ma, S.; Huang, Z.; Karpathy, A.; Khosla, A.; Bernstein, M.; et al. ImageNet Large Scale Visual Recognition Challenge. Int. J. Comput. Vis. 2015, 115, 211-252. [CrossRef]

43. Tufail, A.B.; Ma, Y.K.; Zhang, Q.N. Binary Classification of Alzheimer's Disease Using sMRI Imaging Modality and Deep Learning. J. Digit. Imaging 2020, 33, 1073-1090. [CrossRef]

44. Bereciartua, A.; Picon, A.; Galdran, A.; Iriondo, P. 3D active surfaces for liver segmentation in multisequence MRI images. Comput. Methods Programs Biomed. 2016, 132, 149-160. [CrossRef]

45. He, K.; Zhang, X.; Ren, S.; Sun, J. Deep residual learning for image recognition. In Proceedings of the IEEE Computer Society Conference on Computer Vision and Pattern Recognition, Las Vegas, NV, USA, 27-30 June 2016; IEEE Computer Society: Washington, DC, USA, 2016; pp. 770-778.

46. Szegedy, C.; Vanhoucke, V.; Ioffe, S.; Shlens, J.; Wojna, Z. Rethinking the Inception Architecture for Computer Vision. In Proceedings of the IEEE Computer Society Conference on Computer Vision and Pattern Recognition, Las Vegas, NV, USA, 27-30 June 2016; IEEE Computer Society: Washington, DC, USA, 2016; pp. 2818-2826.

47. Chollet, F. Xception: Deep learning with depthwise separable convolutions. In Proceedings of the 30th IEEE Conference on Computer Vision and Pattern Recognition, CVPR 2017, Honolulu, Hawaii, 21-26 July 2016; Institute of Electrical and Electronics Engineers Inc.: New York, NY, USA, 2017; pp. 1800-1807.

48. Picon, A.; Seitz, M.; Alvarez-Gila, A.; Mohnke, P.; Ortiz-Barredo, A.; Echazarra, J. Crop conditional Convolutional Neural Networks for massive multi-crop plant disease classification over cell phone acquired images taken on real field conditions. Comput. Electron. Agric. 2019, 167, 105093. [CrossRef]

49. Smith, L.N. Cyclical Learning Rates for Training Neural Networks. In Proceedings of the 2017 IEEE Winter Conference on Applications of Computer Vision (WACV), Santa Rosa, CA, USA, 24-31 March 2017; Volume 2015, pp. 464-472.

50. Ioffe, S.; Szegedy, C. Batch normalization: Accelerating deep network training by reducing internal covariate shift. In Proceedings of the 32nd International Conference on Machine Learning, ICML 2015, Lille, France, 6-11 July 2015; International Machine Learning Society (IMLS): Princeton, NJ, USA, 2015; Volume 1, pp. 448-456.

51. Heaven, D. Why deep-learning AIs are so easy to fool. Nature 2019, 574, 163-166. [CrossRef]

52. Kwon, H.; Yoon, H.; Park, K.-W. Multi-Targeted Backdoor: Indentifying Backdoor Attack for Multiple Deep Neural Networks. IEICE Trans. Inf. Syst. 2020, E103.D, 883-887. [CrossRef]

53. Krcmar, H. Informationsmanagement; Springer: Berlin/Heidelberg, Germany, 2015. 
54. Saltz, J.; Hotz, N.; Wild, D.; Stirling, K. Exploring project management methodologies used within data science teams. In Proceedings of the Americas Conference on Information Systems 2018: Digital Disruption, AMCIS 2018, New Orleans, LA, USA, 16 August 2018; Association for Information Systems: Atlanta, GA, USA, 2018.

55. Wirth, R.; Wirth, R. CRISP-DM: Towards a standard process model for data mining. In Proceedings of the Fourth International Conference on the Practical Application of Knowledge Discovery and Data Mining, Manchester, UK, 11-13 April 2000; pp. 29-39.

56. Mcqueen, J.; Meilă, M.; Vanderplas, J.; Zhang, Z. Megaman: Scalable Manifold Learning in Python. J. Mach. Learn. Res. 2016, 17, 1-5. [CrossRef]

57. Breck, E.; Cai, S.; Nielsen, E.; Salib, M.; Sculley, D. The ML test score: A rubric for ML production readiness and technical debt reduction. In Proceedings of the 2017 IEEE International Conference on Big Data, Big Data 2017, Boston, MA, USA, 11-14 December 2017; Institute of Electrical and Electronics Engineers Inc.: New York, NY, USA, 2018; Volume 2018, pp. 1123-1132.

58. Mehrabi, N.; Morstatter, F.; Saxena, N.; Lerman, K.; Galstyan, A. A Survey on Bias and Fairness in Machine Learning. arXiv 2019, arXiv:1908.09635.

59. Biessmann, F.; Salinas, D.; Schelter, S.; Schmidt, P.; Lange, D. Deep learning for missing value imputation in tables with nonnumerical data. In Proceedings of the International Conference on Information and Knowledge Management, Torino, Italy, 22-26 October 2018; Association for Computing Machinery: New York, NY, USA, 2018; pp. 2017-2026.

60. Cox, R.W. AFNI: Software for analysis and visualization of functional magnetic resonance neuroimages. Comput. Biomed. Res. 1996, 29, 162-173. [CrossRef] [PubMed]

61. Jenkinson, M.; Beckmann, C.F.; Behrens, T.E.J.; Woolrich, M.W.; Smith, S.M. FSL. Neuroimage 2012, 62, 782-790. [CrossRef] [PubMed]

62. Jenkinson, M. BET2: MR-Based Estimation of Brain, Skull and Scalp Surfaces. In Proceedings of the Eleventh Annual Meeting of the Organization for Human Brain Mapping, Toronto, ON, Canada, 12-16 June 2005. 